\title{
5G Technology in Internet of Everything (IoE) Application using Light Fidelity (Li-Fi) Indoor Communication
}

\author{
Arba'iah Inn ${ }^{1}$, Rosilah Hassan ${ }^{2}$, Azana Hafizah Mohd $\mathrm{Aman}^{3}$, Liza Abdul Lattif ${ }^{4}$ \\ \{P99946@siswa.ukm.edu.my ${ }^{1}$, rosilah@ukm.edu.my ${ }^{2}$, azana@ukm.edu.my ${ }^{3}$, \\ liza.kl@utm.my $\left.{ }^{4}\right\}$
}

\begin{abstract}
Center for Cyber Security, Faculty of Information Science and Technology, Universiti Kebangsaan Malaysia (UKM), Bangi 43600, Malaysia ${ }^{1}$, Ubiquitous Broadband Access Network Research Group, Universiti Teknologi Malaysia(UTM), 54100 Kuala Lumpur ${ }^{234}$
\end{abstract}

\begin{abstract}
Radio Frequency (RF) spectrum for wireless communication become saturated with the increasing number of smart devices and the demand for wireless data traffic. New wireless communication technologies are needed to fulfill the demand and considered to increase the performance of the system and overcome the bandwidth limitation. Studies have been done that shows light-fidelity ( $\mathrm{Li}-\mathrm{Fi})$ is one of the most promising technology in future which is based on visble light communications (VLC). Initial study on the Li-Fi focuses on achieving speed, security issues, bi-directional concept and support user mobility and multiuser access. This work is hope to complement current work and hence, the objective of this study is to investigate the limitation in Wi-Fi and solve it using $\mathrm{Li}-\mathrm{Fi}$, focusing on indoor mobility and handover. The proposed method will be evaluated using mathematical and algorithm based on the handover proces and the metrics are packet loss and delay.
\end{abstract}

Keywords : Internet of Everything, Light Fidelity (Li-Fi), Visible Light Communication (VLC), mobility, handover.

\section{Introduction}

Light Fidelity (Li-Fi) technology is a new method of data transmission through illumination. The data will be sent using a light emitting diodes (LEDs) and laser diodes with varying intensity faster than human eye can follow. It use a visible light spectrum and infrared for high speed data rate communication. The principle of Li-Fi is by varying the current through light emitting diodes (LED) at very high speed and the output will increase. This concept was founded in 2011 by German Professor Harald Haas, University of Edinburg [1]. It is based on light instead of radio frequency where data for laptops and other mobile devices is transmitted through a light in a room. $\mathrm{Li}-\mathrm{Fi}$ is cheaper then Wi-Fi since visible light spectrum is a free spectrum band. Li-Fi is the future $5^{\text {th }}$ Generation $(5 \mathrm{G})$ mobile network [2].

The revolution of the Internet of Things (IoT) makes all devices and sensor capable to connect through all the networks and with less human interaction. It enhances the productivity of the industry through high level of automation and Industrial Internet of Things (IIOT). This was led to the innovations for the small factory which increases the speed and the quality of the product by using the sensor and machine to machine communications. This positive growth 
will be a good investment for the industries and accept the Fourth Industrial Revolution (4IR) technologies. IoE extends the IoT technology which is more complex in how device to device communicate. With Li-Fi technology, 5G network will still become the backbone to fullfill all the IoE devices development [3], as shown in Figure 1.

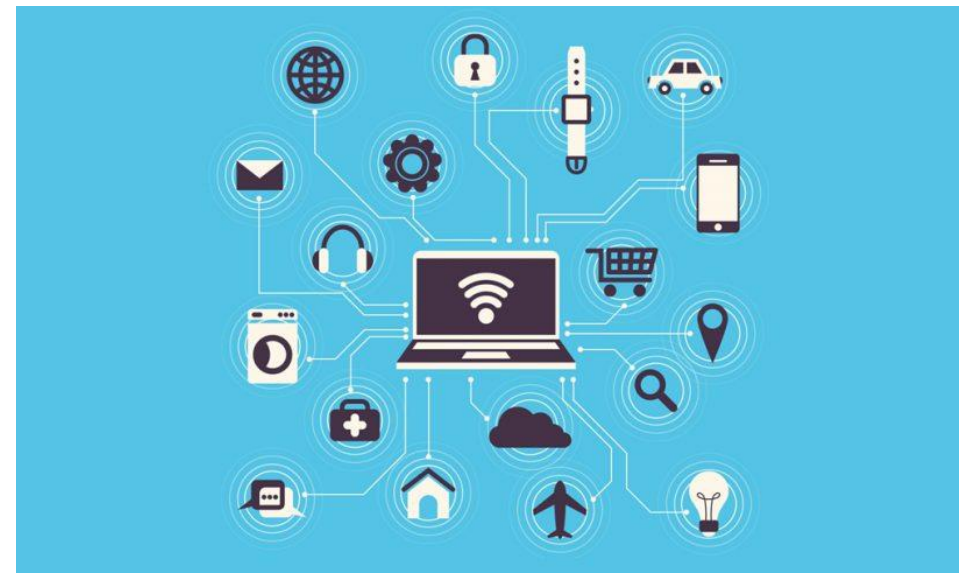

Figure 1. 5G network backbone with IoE devices

IoE is an application which can benefit from a Li-Fi application since energy utilization could be as low as possible [4] [5]. It develops from the concept for the Internet where all things around us are connected to the internet and has the capability to exchange information, organize data and work in all circumstances. Research on Li-Fi for future 5G technology can be focused on energy consumption and handover management purpose which can solve the demand of high traffic data.

The convergence of hardware, software and services will enable novel integrated healthcare solution and it can offer a great opportunity for different consumers including organizations, governments, companies or even the end user. Also for the business, IoE can provide systems that have the ability to automate automated manufacturing, automate public transportations and other tasks [6].

The aim of this research is to use the Li-Fi technology in the IoE environment with several approaches and frameworks and will focus on handover and mobility. Li-Fi technology is faster and secured and it will avoid the dependency for radio frequency. It also can avoid the spectrum issue and save energy.

The multiple section of this paper are organized as follows, section 2 will give an overview of $\mathrm{Li}-\mathrm{Fi}$ for indoor and handover communications. In Section 3, the Li-Fi concept and characteristis is described. In section 4 the research methodology is explained and Section 5 discusses the conclusion and future research.

\section{Overview of Li-Fi Technology}

The rapid development of wireless communication in healthcare application, smart home application offers many challenges such as handover process, reliable data transmission, and 
time delivery of data, security and power management [7]. Li-Fi can easily be used in all places where Bluetooth, infrared, Wi-Fi and internet are difficult to cover especially in rural area and the issue of the shortage of radio frequency bandwidth can be overcome by using $\mathrm{Li}-\mathrm{Fi}$ Technology. For the handover performance in Li-Fi networks, there are number of studies in published research. The improvement throughput and delay in bidirectional transmission [8] and the handover modelling for indoor Li-Fi [9].

\subsection{Indoor mobility and handover communications}

The study will focus on the indoor scenario, looking on the time taken for the bidirectional communication from the Access Point to the user (downlink) and from the user to the Access Point (uplink). The aim for indoor communication is to achieve higher data rates and reduce the delay between the uplink and downlink communication. The comparative study will study the on-going modulation and coding technique and propose new modulation method which is suitable in optical wireless communication. Based on the propose method and solution, the mathematical model and algorithms will be developed and propose the evaluation measurement to get the comparison result. Figure 2 show the concept of Li-Fi technology which consists of Access Point 1 (AP1) and Access Point 2 (AP2). Communication between user 1 with Access Point 1, and user 1 with Access Point 2 is peer to peer communication. Handover process will take part when user 1 move into Access Point 2 coverage. Handover basically means changing the Access Point of connection while communicating without losing the connection. Research will focus on the time taken or the delay between the uplink and downlink in peer to peer communication and also in the handover process from Access Point 1 to Access Point 2.

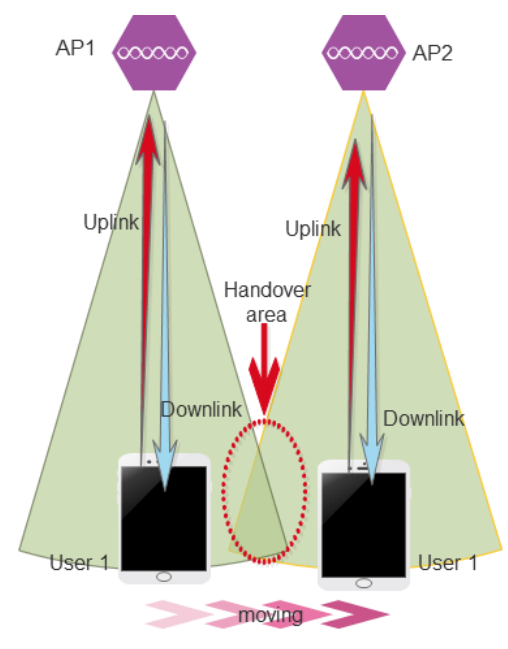

Figure 2. Li-Fi uplink and downlink transmission 


\section{Concept of $\mathrm{Li}-\mathrm{Fi}$}

The sending of data in Li-Fi technology is based on fibre optic communication concept, when the data is sent the light will switch "ON' and "OFF". Li-Fi can work in three mode which are peer to peer topology or line of sight communication, star topology and broadcast communication. However, some study shows that there is some challenge to look on how the receiver will transmit back the data to the transmitter [10]. Table 1 shows the comparison between Li-Fi technology and the Wi-Fi technology [11].

Table 1. Comparison between Li-Fi and Wi-Fi technologies.

\begin{tabular}{|c|c|c|}
\hline & Li-Fi & Wi-Fi \\
\hline Speed & $1-3.5 \mathrm{Gbps}$ & 54-250 Mbps \\
\hline Range & $\begin{array}{l}10 \text { meters/ Based on LED } \\
\text { light }\end{array}$ & 20-100 meters \\
\hline IEEE Standard & 802.15 .7 & $802.11 b$ \\
\hline Network & Point-to-point & Point-to- \\
\hline $\begin{array}{l}\text { Topology } \\
\text { Data Transfer }\end{array}$ & Use light as a carrier & $\begin{array}{l}\text { Multipoint } \\
\text { Use radio spectrum }\end{array}$ \\
\hline Medium & & \\
\hline Frequency Band & 100 times of $\mathrm{THz}$ & $2.4 \mathrm{GHz}$ \\
\hline Security & High secure & Medium secure \\
\hline Devices & $\begin{array}{l}\text { Mobile phones., office and } \\
\text { industrial automatic devices, } \\
\text { notebook computers, sever } \\
\text { and TV }\end{array}$ & $\begin{array}{l}\text { Notebook } \\
\text { computers, desktop } \\
\text { computers, servers, } \\
\text { TV }\end{array}$ \\
\hline Users & $\begin{array}{l}\text { Home users, public users, } \\
\text { travelling users, office and } \\
\text { industrial workers, aircraft } \\
\text { cabins, hospitals. }\end{array}$ & $\begin{array}{l}\text { Corporate, campus } \\
\text { users, homes and } \\
\text { other public places. }\end{array}$ \\
\hline $\begin{array}{l}\text { Development } \\
\text { started }\end{array}$ & 2011 & 1990 \\
\hline
\end{tabular}

Visible light communication is based on IEEE standard 802.25.7 [11] with data rates from $11.67 \mathrm{~kb} / \mathrm{s}$ to $96 \mathrm{Mb} / \mathrm{s}$ for indoor and outdoor environment. It can use three topologies which are peer to peer, star topology and broadcast topology. Table 2 show the indoor and outdoor application. 
Table 2 Li-Fi indoor and outdoor characteristics

\begin{tabular}{|c|c|c|c|}
\hline Author's/ Year & Application & Indoor & Outdoor \\
\hline Rajagopal, S., & Modulation type & On Off Keying,OOK & On Off Keying, OOK \\
\hline Roberts, R. D., \& & & Variable Pulse Position & Variable Pulse Position \\
\hline Lim, S. K. (2012) & & Modulation,(VPPM) & Modulation,(VPPM) \\
\hline \multirow{7}{*}{$\begin{array}{l}\text { Kuppusamy, P., } \\
\text { Muthuraj, S., \& } \\
\text { Gopinath, S. } \\
(2016)\end{array}$} & Speed, Mbit/s & $1.25-96$ & $0.012-0.268$ \\
\hline & Topology & Peer to peer & Star topology \\
\hline & & & Broadcast \\
\hline & Coding & Reed Solomon(RS) & Reed Solomon(RS) \\
\hline & & & $\begin{array}{l}\text { Convolutional } \\
\text { Codes }(C C)\end{array}$ \\
\hline & Potential & Fluorescent light & Day light \\
\hline & Interference & & Fluorescent light \\
\hline
\end{tabular}

\section{Research Methodology}

Future developments, $\mathrm{Li}-\mathrm{Fi}$ is an efficient alternative to radio based wireless communication where every bulb can be used as a Access Point (AP). This may solve issue such as shortage of radio frequency bandwidth. The move from point to point links to full wireless network based on light have several challenges by considering on the received signal intensity and the limited field of view for every bulb. Within each access point, there can be several users and therefore multiple access technique are required and based on the uplink and downlink approach [12].Within each access point we can study on the co-channel interference where two access point in adjacent area will interfere with each other.

\section{Conclusion and Future Work}

Li-Fi technology provides the potential for high data rates communication for indoor and outdoor application. The technology use at low power and cost by using LEDs as transmitter and Photodector as receiver. With the generation of LEDs nano, data can be sent at a very high data rates. Li-Fi technology is a complementry to Wi-Fi for $5 \mathrm{G}$ technology, safer in some area like in petrol pump and hospitals, no license needed and it is a green technology by using light instead of radio wave. In this paper, we study the handover process for indoor and outdoor environment for the optimization of access point sharing and minimize the handover failure in multiuser.

Future research, we will look on the outdoor implementation because of rapid increase in $\mathrm{Li}-\mathrm{Fi}$ technology using light infrastructures as future high data rate communications. Indeed more study and research will be done on modulation method, algorithms by using the same mathematical and model propose in the indoor scenario. This paper is an ongoing study of our related research for indoor communications focusing on uplink and downlink communication and also handover process in Li-Fi technology. 
Acknowledgements. We would like to acknowledge the discussion and assistance provided by the Network and Communication Technology Research Group, FSTM, UKM and Ubiquitous Broadband Access Network Research Group, UTMKL.

\section{References}

[1] H.Haas, L.Yin, L.Wang,C. Chen . : "What is LiFi?” IEEE J.Light Technol.,34(6), pp 15331544 (2016)

[2] J.Khurpade, D.Rao,P.Sanghavi, : "A Survey on IOT and 5G Network", International Conference on Smart City and Emerging Technology,ICSCET 2, pp 1-3 (2018)

[3] Sensor and Actuators A: Physical, Volume 272,pp 187-198 (2018).

[4] E.Pikasis \& W.O.Popoola, : "Understanding LiFi Effect on LED Light Quality", IEEE Communication (2018)

[5] I.Tavakkolnia,C.Cheng,R.Bian \& H.Haas, : " Energy-Efficient Adaptive MIMO-VLC Technique for Indoor LiFi Application”, IEEE Communications, 331-335 (2018)

[6] Madakam, S., Ramaswamy, R., \& Tripathi, S. : Internet of Things (IoT): A literature review. Journal of Computer and Communications, 3(05), 164 (2015)

[7] Waluyo A.b., Pek.I.,Chen.X., Yeoh W.S. : "Design and Evaluation of Lightweight Middleware for Personal Area Networks. Pers. Ubiquit. Comput.; 509-525. (2009)

[8] H.Alshaer, H.Haas, : "Bidirectional LiFi Attocell Access Point Slicing Scheme",IEEE Transactions on Network and Service Management, Vol. 15,No. 3, (2018).

[9] M. Dehghani Soltani, H.Kazemi, M. Safari, H.Haas, : "Handover Modelling for Indoor LiFi Cellular Networks: The Effects of Receiver Mobility and Rotation",Li-Fi R\&D Centre, Institute for Digital Communications, University of Edinburgh, UK. (2017)

[10] S.Chatterjee.,S.Agarwal.,A.Nath., : "Scope and challenges in Light Fidelity(LiFi) Technology in Wireless Data Communication". InternationalJournal of (2015).

[11] S.Rajagopal,R.D.Roberts, S.Lim, : " IEEE 802.15.7 Visible LIGHT Communication: Modulation Schemes and Dimming Support”, IEEE Communications Magazine (2012).

[12] M. Ayyash. : "Coexistence of $\mathrm{WiFi}$ and $\mathrm{LiFi}$ toward 5G:concepts,opportunities and challenges”. IEEE Commun. Mag. ,54(2),pp 64-71(2016)

[13] Alsubaie,S.M.s.,Othman,N.E.,\& Hassan,R. : "Enhancement of geometrical predictive handover probability based on coverage sectors". Journal of Theoritical and Applied Information Technology, 96(16), 5291-5302,(2018)

[14] Saadi,Y.A.,Hassan,R., \& Dahlila Putri, D.S, : ”Energy-aware for DH-AODV routing protocol in Wireless Mesh Network".ARPN Journal of Engineering and Applied Sciences, 12(13), 4122-4128. (2006)

[15] Hassan, R., Jubair,A.M., Azmi,K.,\& Abu Bakar,K.(2016), : ”Adaptive congestion control mechanism in CoAP Application Protocol for Internet of Things (IoT). International Conference on Signal Processing and Communication, ICSC ., pp 121-125. (2016). 